\title{
Polyglycerol Modified Polysiloxane Surfactants :Their Adsorption and Aggregation Behavior in Aqueous Solution
}

Guoyong Wang ${ }^{\mathrm{a}, *}$, Yanyan Zhu ${ }^{\mathrm{b}}$, Yunhui Zhai ${ }^{\mathrm{c}, *}$, Wanxu Wang ${ }^{\mathrm{a}}$, Zhiping Du ${ }^{\mathrm{a}}$, Jieqiong Qin ${ }^{\mathrm{a}}$ 
A simple process to synthesize polyglycerol modified polysiloxane surfactants has been carried out applying a "grafting-to" approach. After formation of allyl polyglycerol ether (APGE) from diglycerol (DGY) and allyl glycidyl ether (AGE), polysiloxane modified by designed number of polyglycerol groups (PHMS-APGE) were obtained by connecting the APGE structure to different $\mathrm{Si}-\mathrm{H}$ functionalized polyhydromethyl siloxane (PHMS). The synthesized APGE-PHMS were characterized by IR and ${ }^{1} \mathrm{H}$ NMR. Solubility measurements showed that the solubility of the as-prepared polymers increased in polar solvents with the increasing of the amount of hydrophilic APGE groups. Furthermore, the surface activity, surface adsorption, aggregation and spreading behaviors of APGE-PHMS were investigated utilizing surface tension measurements, dynamic light scattering (DLS), transmission electron microscopy (TEM) and contact angle measurements. The results showed that critical aggregation concentration (CAC) and the time needed to reach adsorption equilibrium on water surface decrease systematically with the increase in the number of APGE groups. These polymers in aqueous solutions could aggregate to form spherical assemblies. Moreover, the spreading behavior of these polymers on low energy surface was found to be influenced by the number of hydrophilic APGE groups.

Keywords: Polysiloxane; Polyglycerol; Adsorption behavior; Aggregation behavior

\section{Graphic Abstract:}

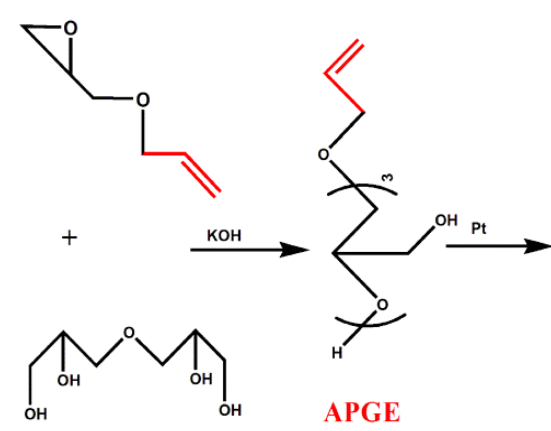

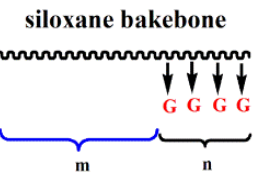

(1) $m=8, n=2$

(2) $m=6, n=4$

(3) $m=5, n=5$

(4) $m=4, n=6$

(5) $\mathrm{m}=2, \mathrm{n}=8$

APGE-PHMS 


\section{Introduction}

Silicones have drawn people's attention for many years due to their unique properties. For instance they exhibit constant viscosity over a broad temperature range as well as an extremely high gas permeability and absolutely bio-inert. Thus, silicones are widely used in cosmetics, medicine, coatings, electronics and textiles, and so on[1]. Nevertheless, the hydrophobicity, which is the most significant feature of silicone, has always been a drawback for a great number of applications[2, 3]. Therefore, current attempts mostly focus on combination of silicones and hydrophilic materials in order to combine the unique silicone-specific properties with hydrophilicity. Stadler et al. focused on the attachment of various kinds of oligo- and polysaccharides[4-6]. Paul et al. synthesized hydrophilic silicone with glycerol side chains[2]. Further information on polymers combining silicone with various other hydrophilic groups, either in the form of random or block copolymers or with grafted[7], branched[8-10], star-shaped[11], or cross-linked[12-14] polymers, has been reported. Polyether modified polysiloxane is one of the most popular hydrophilic silicones. The specific properties of polyether modified polysiloxane have also been studied in a lot of publications[15-16]. However, the raw material of polyether may contain carcinogenic 1,4-dioxane, which is banned in some personal care products[17]. At the same time, the raw material for polyether, which depends on fossil fuels, is not sustainable.

Glycerol is water-soluble and renewable. Moreover, with the development of biodiesel, surplus glycerol has become an urgent issue. But little research has been 
done on combination of silicone and hydrophilic polyglycerol. Here we report a two-step synthetic approach to graft polyglycerol groups to the polysiloxane backbone[18]. We further investigated their basic properties and application performances.

\section{Experimental}

\subsection{Materials}

Diglycerol (DGY, 81\%) and allyl glycidyl ether (AGE, 99\%) were purchased from TCI. Potassium hydroxide (KOH, 99\%) was supplied by Tianjin Chemical Reagent Co., Ltd. (China). Hexachloroplatinic acid $\left(\mathrm{H}_{2} \mathrm{PtCl}_{6} \cdot 6 \mathrm{H}_{2} \mathrm{O}, 99.9 \%\right)$ was provided by Nanjing Chemical Reagent Co., Ltd. (China). Isopropanol (IPA, 99.8\%) and sulfuric acid $\left(\mathrm{H}_{2} \mathrm{SO}_{4}, 98 \%\right)$ were purchased from Beijing chemical Reagent Co., Ltd. (China). Other reagents, i.e., including octamethyl cyclotetrasiloxane $\left(\mathrm{D}_{4}\right)$, poly(methylhydrosiloxane) (Mw 3792) and hexamethyldisiloxane (MM) were of technical grade and obtained from Zhejiang Runhe Organosilicon New Material Co., Ltd. (China). Double distilled water was used for analyses and property measurements. All commercially available chemicals were used as received without any further purification.

\subsection{Synthesis of Polyglycerol-modified Polysiloxane (APGE-PHMS)}

2.2.1 Synthesis of Allyl Polyglycerol Ether (APGE) and Polyhydromethyl Siloxane (PHMS)

Under $\mathrm{N}_{2}, 19.94 \mathrm{~g}(0.12 \mathrm{~mol})$ of diglycerol was reacted with $11.42 \mathrm{~g}(0.10 \mathrm{~mol})$ of allyl glycidyl ether in the presence of potassium hydroxide as catalyst and the mixture was stirred for $3 \mathrm{~h}$ at a reflux temperature of $90^{\circ} \mathrm{C}$. Then the product was 
neutralized by adding $5.00 \mathrm{~g}$ of cation exchange resin and stirring for further 20 minutes, followed by filtration at $70^{\circ} \mathrm{C}$. The volatile matters in the filtrate were removed by stripping for $2 \mathrm{~h}$ at $60^{\circ} \mathrm{C}$ under a pressure of $5 \mathrm{mmHg}$. A light yellow liquid product (APGE) was obtained in $95 \%$ yield.

Polyhydromethyl siloxane (PHMS) were prepared with $\mathrm{D}_{4}$, poly(methylhydrosiloxane) (Mw 3792) and hexamethyldisiloxane (MM) catalyzed by sulfuric acid. The method has already been reported in literature [18]. Thus, PHMS with 2, 4, 5, 6 and $8 \mathrm{Si}-\mathrm{H}$ bonds according to feed composition were obtained in $90 \%$ yield (Table 1).

Table 1 Composition of PHMS and their molecular weights

\begin{tabular}{ccccccc}
\hline $\begin{array}{c}\text { Sample } \\
\text { number }\end{array}$ & Feed composition & $\mathrm{n}$ & $\begin{array}{c}\text { Composition } \\
(\mathrm{g} / \mathrm{mol})\end{array}$ & $\begin{array}{c}\text { Mn } \\
\left({ }^{29} \text { Si-NMR }\right)\end{array}$ & $\begin{array}{c}{ }^{29} \text { Si-NMR } \\
\text { spectra } \\
(\mathrm{g} / \mathrm{mol})\end{array}$ \\
\hline 1 & 8 & 2 & 874 & 8.38 & 1.49 & 872 \\
2 & 6 & 4 & 846 & 5.76 & 3.15 & 777 \\
3 & 5 & 5 & 832 & 4.82 & 4.10 & 765 \\
4 & 4 & 6 & 818 & 4.24 & 6.25 & 851 \\
5 & 2 & 8 & 790 & 1.70 & 7.83 & 758 \\
\hline
\end{tabular}

\subsubsection{Synthesis of Polyglycerol-modified Polysiloxane (APGE-PHMS)}

Polyglycerol-modified polysiloxane (APGE-PHMS) were synthesized by the hydrosilylation reaction from APGE and PHMS with different Si-H groups. Five sets of APGE-PHMS systems were synthesized and the number of APGE contents was 
expected to be 2, 4, 5, 6 and 8 respectively. For example, APGE(5)-PHMS was synthesized as follow. In a $250 \mathrm{ml}$ three-necked round bottom flask, $26.2 \mathrm{~g}(0.08 \mathrm{~mol})$ APGE together with $14.9 \mathrm{~g}$ (0.08 mol, referring to the amount of Si-H-groups) PHMS $(m=5, n=5)$ were dissolved in isopropanol. The mixture was heated to to reflux under nitrogen atmosphere. The reaction was catalyzed by chloroplatinic acid. After 6 hours, IR spectra showed complete disappearance of the $\mathrm{Si}-\mathrm{H}$ bond. After completion of the reaction, the product was stripped at $60^{\circ} \mathrm{C}$ under a pressure of $5 \mathrm{mmHg}$ to remove the volatile matters. A light yellow liquid product (APGE-PHMS) was obtained in 90\% yield.

\subsection{Characterization of structures}

FT-IR spectra were measured using a Bruker V70 Fourier transform spectrometer (Germany) with a $\mathrm{KBr}$ plate. ${ }^{1} \mathrm{H}$ NMR and ${ }^{29} \mathrm{Si} \mathrm{MNR}$ spectras were conducted with Bruker-400 MHz spectroscopy (Germany) at room temperature.

\subsection{Measurement of solubility}

Solubility was measured by dissolving APGE-PHMS directly in different solvents of various polarities at a concentration of $100 \mathrm{mg} / \mathrm{mL}$ at room temperature. There is no recognized standard to judge the polarity of solvent. We usually determine the polarity of the solvent according to the solvent dielectric constant. Thus, we choose seven solvents (isooctane, ether, methylene chloride, isopropanol, acetonitrile, dimethylsulfoxide, water) covering dielectric constant from 2 to 80 to carry out the experiment. 


\subsection{Measurement of equilibrium surface tension}

The equilibrium surface tension of APGE-PHMS solutions was measured using a Krüss K12 Processor Tensiometer (Germany) and determined by the Wilhelmy plate method. The APGE-PHMS solutions were prepared by direct dissolution of APGE-PHMS in double distilled water. The length and thickness of the platinum plate were $19.9 \mathrm{~mm}$ and $0.2 \mathrm{~mm}$, respectively. The dipping distance was $2 \mathrm{~mm}$. The surface tension of the double distilled water was confirmed as being in the range of $72.0 \pm 0.3$ $\mathrm{mN} / \mathrm{m}$ at $25.0 \pm 0.1{ }^{\circ} \mathrm{C}$, prior to measurements. The surface tension of each concentration was measured three times with an average deviation less than $0.2 \mathrm{mN} / \mathrm{m}$ at intervals of $90 \mathrm{~s}$ after stirring.

\subsection{Measurement of dynamic surface tension}

The dynamic surface tension was measured by a Krüss BP100 bubble-pressure Tensiometer (Germany). The solutions were also prepared by dissolving APGE-PHMS directly in double distilled water. This measurement was conducted with effective surface ages ranging from 10 to $200,000 \mathrm{~ms}$.

\subsection{Measurement of aggregation behavior}

The aggregation behavior of APGE-PHMS solutions was monitored by dynamic light scattering (DLS) with a Zeta Plus Particle Size Analyzer (Brookhaven, USA) and the scattering angle was set at $90^{\circ}$. All of the solutions were filtered with a 0.45 $\mu \mathrm{m}$ (mixed cellulose acetate) membrane filter.

The structures of APGE-PHMS aggregates in solutions were also observed with a JEM-1011 (JEOL Co., Japan) transmission electron microscopy (TEM) by negative 
staining. A droplet of the surfactant solution was added onto a carbon-coated grid and allowed to equilibrate for $2 \mathrm{~min}$. The removal of excess liquid was performed by blotting carefully with a filter paper. When the grid was partially dried, a drop of staining solution ( $2 \%$ phosphotungstic acid) was added onto the grid. Again, the removal of excess liquid was performed after $2 \mathrm{~min}$, and the grid was dried at room temperature.

\subsection{Measurement of spreading ability}

The spreading ability of prepared surfactant solutions over hydrophobic substrates was measured using drop shape analyzer DAS-100 (Krüss Company, Germany). Paraffin film was chosen as solid substrates showing different solid-air interfacial energies in terms of contact angles for water $(106 \pm 2)^{\circ}$ on paraffin. The hydrophobic film was placed on an aclinic stage in front of a microscope, which was connected to a $\mathrm{CCD}$ camera. To ensure minimal relative error, every measurement was repeated at least three times. The contact angles were the mean values obtained in each experiment.

\section{Results and discussion}

\subsection{Synthesis of polyglycerol-modified polysiloxane (APGE-PHMS)}

Polyglycerol-modified polysiloxane were synthesized via the hydrosilylation reaction which grafts polyglycerol groups to the polysiloxane backbone. The synthesis included a two-step method as shown in Scheme 1. The first step was to produce the polyglycerol containing end group structure of $\mathrm{C}=\mathrm{C}$ and polysiloxane containing designed number of $\mathrm{Si}-\mathrm{H}$ bonds. In this simple procedure, allyl polyglycerol ether 
was obtained, in which the $\mathrm{C}=\mathrm{C}$ bond could be attached to PHMS with $\mathrm{Si}-\mathrm{H}$ bond. In the second step, five sets of polyglycerol-modified polysiloxane were prepared using APGE and polysiloxane with designed $\mathrm{Si}-\mathrm{H}$ contents, which was catalyzed by chloroplatinic acid[18].

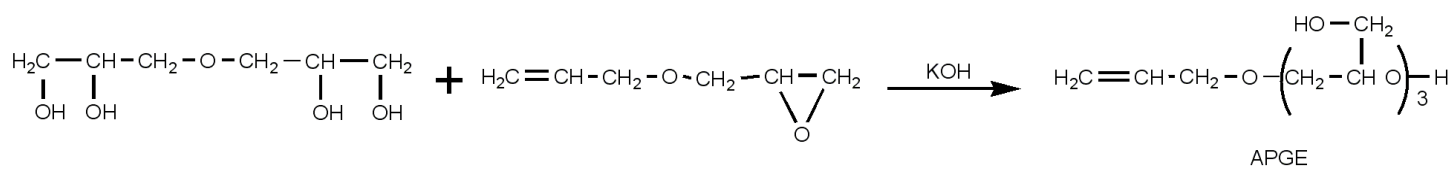

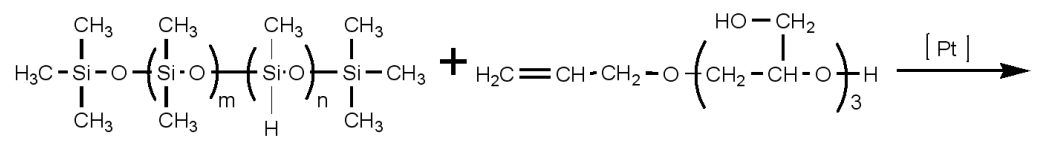

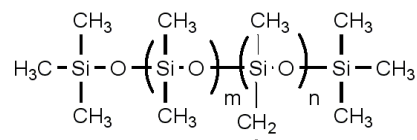

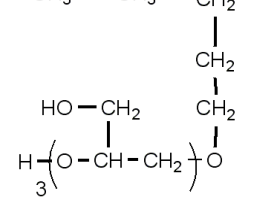

$$
\begin{aligned}
& \text { APGE-PHMS } \\
& \begin{array}{ll}
\text { (1) } m=8, n=2 & \text { (2) } m=6, n=4
\end{array} \\
& \text { (3) } m=5, n=5 \text { (4) } m=4, n=6 \\
& \text { (5) } m=2, n=8
\end{aligned}
$$

Scheme 1. Synthetic route of APGE-PHMS

${ }^{1} \mathrm{H}$ NMR spectra of APGE shows complete disappearance of the typical peak of epoxy group at 2.3-3.1 ppm and the double bond signals of allyl glycidyl ether at 5.2-5.9 ppm appear, confirming the complete reaction of epoxy ring opening (seen in Fig.1). The result of ${ }^{1} \mathrm{H}$ NMR suggested that APGE was obtained successfully. 


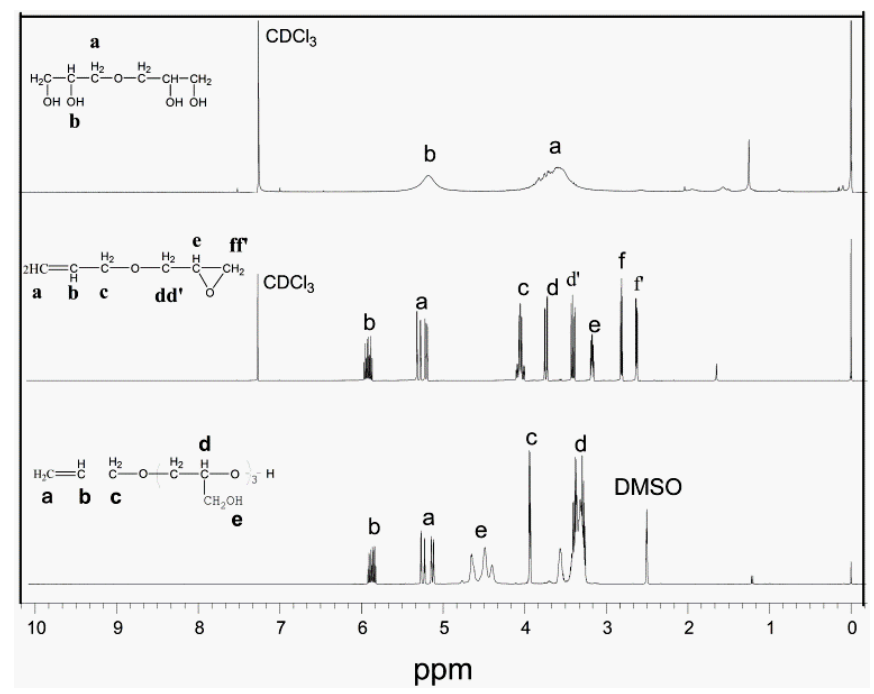

Fig. $1{ }^{1}$ H-NMR spectra of (1) DGY, (2) AGE and (3) APGE

Meanwhile, the ${ }^{29} \mathrm{Si}$-NMR spectrum for PHMS $(\mathrm{m}=5, \mathrm{n}=5)$ is shown in Fig.2 as an example. ${ }^{29} \mathrm{Si}-\mathrm{NMR}$ spectrum confirmed that the five sets of PHMS (Table 1) were basically synthesized as expected. Three typical signals were observed. The characteristic peak of $\mathrm{Si}\left(\mathrm{CH}_{3}\right)_{3}$ could be found at $9.0 \mathrm{ppm}$. The peak at $-37.5 \mathrm{ppm}$ is corresponding to $\mathrm{Si}-\mathrm{H}$. Meanwhile, the peak of $\mathrm{Si}\left(\mathrm{CH}_{3}\right)_{2}$ is observed at -20.0 $\operatorname{ppm}[20]$.

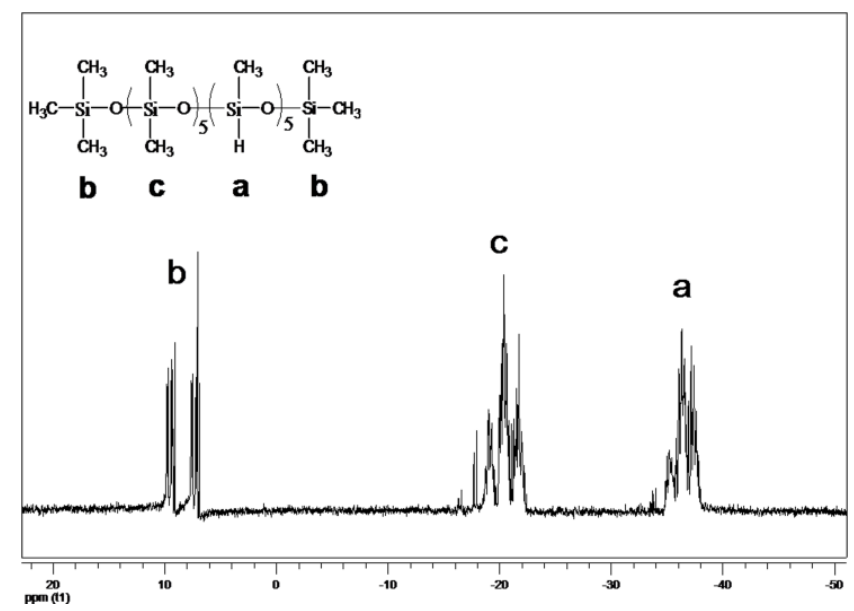

Fig. $2{ }^{29}$ Si-NMR spectra of PHMS

The final products APGE-PHMS were characterized by FT-IR and ${ }^{1} \mathrm{H}-\mathrm{NMR}$. FT-IR and ${ }^{1}$ H-NMR spectra of APGE(5)-PHMS is shown in Fig. 3 for example. The reaction process was monitored by IR spectroscopy (Fig.3), from which the gradual 
disappearance of the peak of Si-H bond at about $2155 \mathrm{~cm}^{-1}$ could be observed [20]. Complete conversion could easily be confirmed by ${ }^{1} \mathrm{H}$ NMR spectroscopy (Fig.4), where the characteristic absorption of neither $\mathrm{Si}-\mathrm{H}$ at $4.8 \mathrm{ppm}$ nor $\mathrm{C}=\mathrm{C}$ of $\mathrm{APGE}$ at 5.2-5.9 ppm could be found in the spectroscopy. Meanwhile, a peak at about $0.5 \mathrm{ppm}$ corresponding to $\mathrm{Si}-\mathrm{CH}_{2}$ arose, which also suggested the termination of hydrosilylation reaction [2]. Both the analysis of FT-IR and ${ }^{1} \mathrm{H}-\mathrm{NMR}$ confirm that APGE-PHMS were successfully obtained.

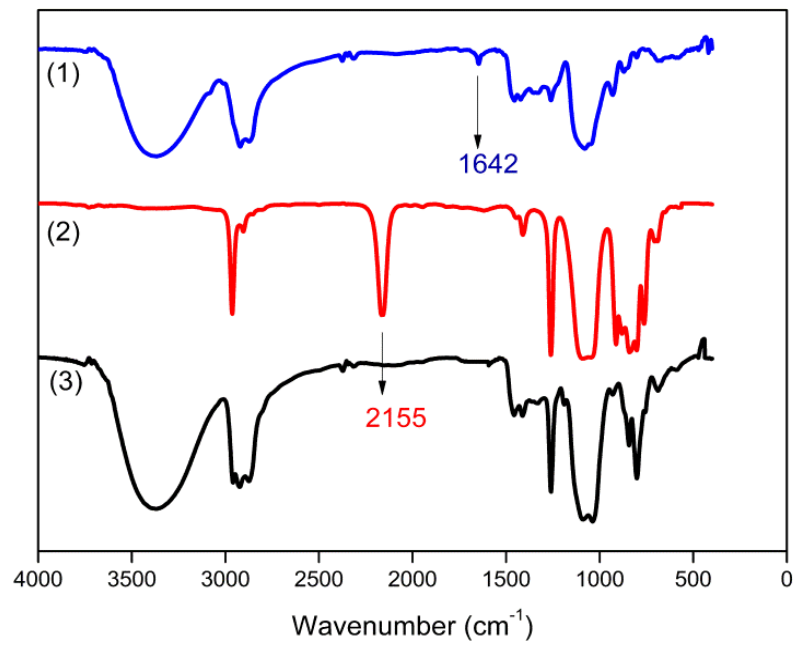

Fig.3 Overlapped FT-IR spectra of (1) APGE, (2) PHMS and (3) APGE-PHMS

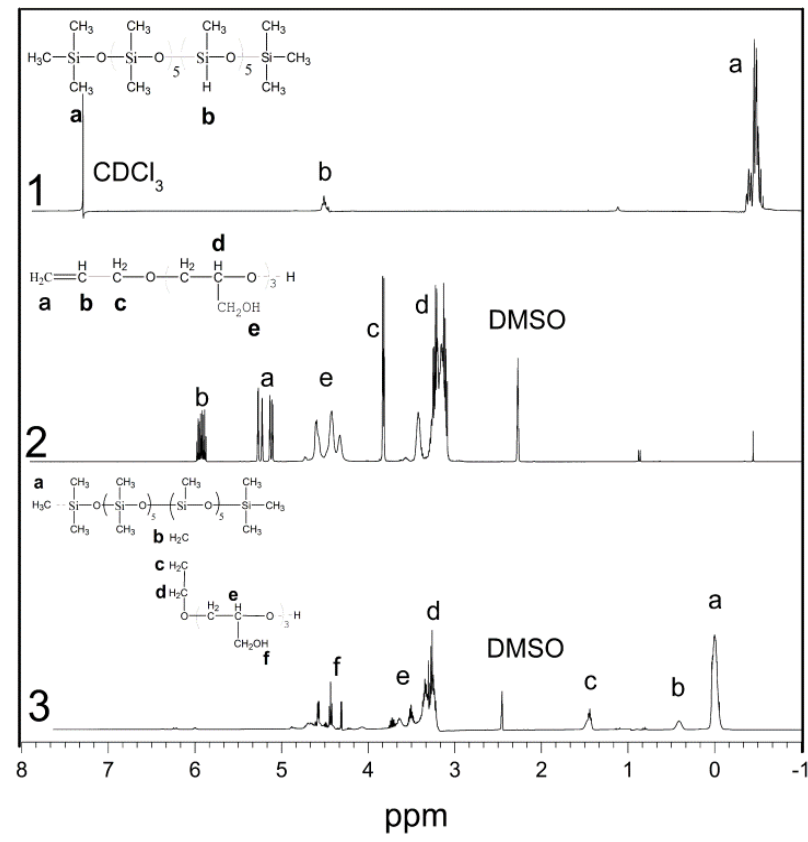




\subsection{Solubility}

The most prominent feature of polysiloxane is their hydrophobicity. Because of the shielding effect of the organic moieties connected to silicone, polysiloxane present insolubility in water or polar organic solvents. Grafting polar groups of APGE to the polysiloxane molecular chains could change polarity and consequently improve solubility in polar solvents. So we take the method of dissolving APGE-PHMS in different solvents of various polarities to quantify their hydrophobicity. The number of APGE groups per mole APGE-PHMS can be used as horizontal ordinate.

Fig.5 shows the result of the experiment of solubility. As we can see, unlike polysiloxane, APGE-PHMS are soluble not only in nonpolar solvents but also polar solvents. The structure of APGE-PHMS can be described as brush-like, with the grafts forming the hydrophilic bristles of the brush.[2] These bristles are able to increase the hydrophilicity of polymer backbone, forming a hydrophilic surface of APGE-PHMS. The density of this hydrophilic surface depends on the number of APGE groups in the polymer. So the more number of APGE groups leads to an increasing solubility in polar solvents. Therefore, APGE-PHMS of various solubilities can specifically be designed. 


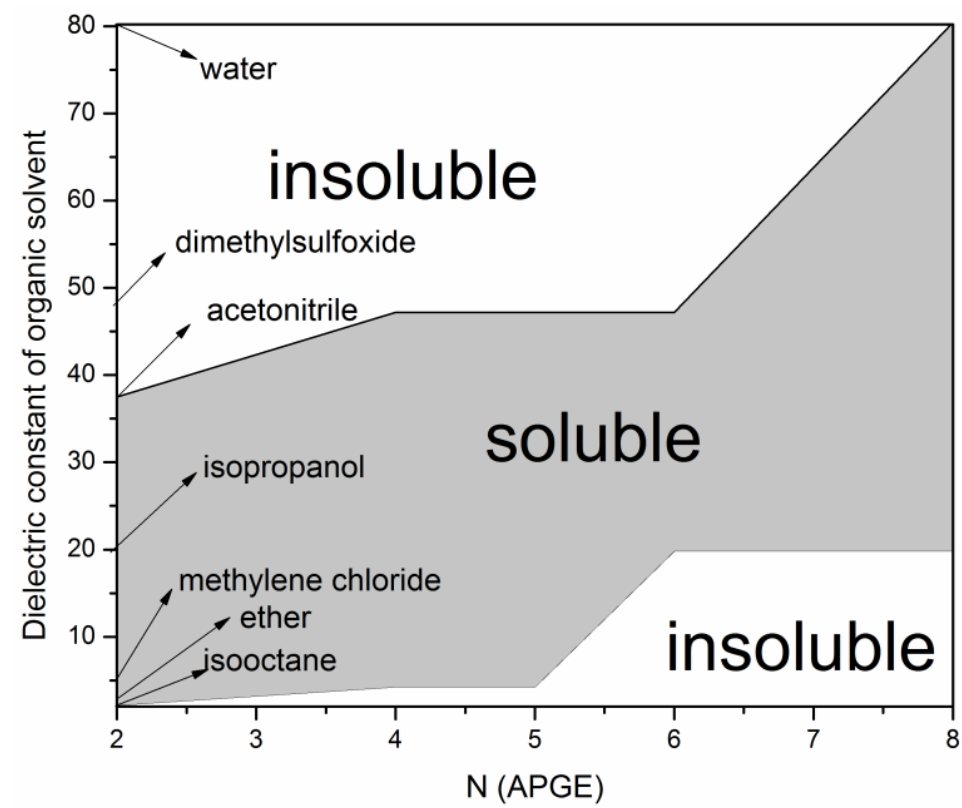

Fig.5 The solubility of the five sets of APGE-PHMS in various solvents of different polarity

\subsection{Surface Activity}

The equilibrium surface tension is the most common way to evaluate the surface activity of surfactants. The surface tension of APGE(2)-PHMS was not measured because of its poor solubility in water at room temperature. In the following research, system 1 was not tested for the same reason. Inspection of the data in Fig. 6 shows that APGE-PHMS reduce the surface tension of water at low concentration, indicating that these APGE-PHMS molecules adsorb strongly at the air/water surface and they are highly effective surfactants. The critical aggregation concentration (CAC) and the surface tension at $\mathrm{CAC}\left(\gamma_{\mathrm{CAC}}\right)$ were also obtained from the breakpoint of equilibrium surface tension-concentration curve. The values of CAC and $\gamma_{\mathrm{CAC}}$ of the four sets were summarized in Table 2. One may discover that the four polymers show two breakpoints in their surface tension curves. The result is in agreement with other siloxane surfactants. This may be attributed to the formation of pre-micellar aggregations before true micelles are formed. Meanwhile, the APGE-PHMS could 
reduce the surface tension of water to $21 \mathrm{mN} / \mathrm{m}$ approximately, which is lower than ordinary hydrocarbon surfactants. The reason of this consequence may be that the methyl groups in polysiloxanes backbone packing at the air/solution interface have an intrinsically lower surface energy than methylene groups of ordinary hydrocarbon surfactants. On the other hand, with an increase in the number of APGE groups in the molecules, the CAC decreases gradually, which is not consistent with the result of other surfactants. Similar observation was shown by Han Fu[22]. The result indicates that CAC may be affected not only by the number, but also by the position of APGE in the molecule.

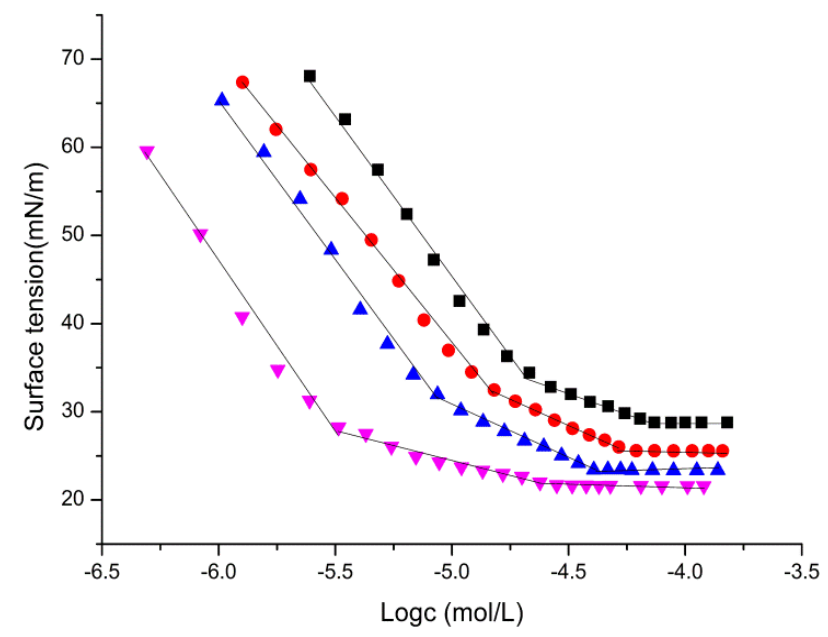

Fig.6 Equilibrium surface tension with concentration for APGE-PHMS at 298K: APGE(4)-PHMS •, APGE(5)-PHMS $\boldsymbol{\Lambda}$, APGE(6)-PHMS $\nabla$, APGE(8)-PHMS

At equilibrium, by calculating the surface excess concentration $\Gamma_{\mathrm{CAC}}$, the area/molecule $A_{\mathrm{CAC}}$, the adsorption efficiency $\Pi_{\mathrm{CAC}}$, the standard free energy of aggregation $\Delta \mathrm{G}^{0}{ }_{\text {mic }}$ and adsorption $\Delta \mathrm{G}_{\text {ads }}^{0}$ as estimated from five equations below (1-5), the packing characteristics of the groups at the interface can be inferred.

$$
\begin{aligned}
& \Gamma_{\mathrm{CAC}}=(-1 / \mathrm{nRT}) /(\mathrm{d} \gamma / \mathrm{d} \operatorname{lnC}) \\
& \mathrm{A}_{\mathrm{CAC}}=10^{16} /\left(\mathrm{N}_{\mathrm{A}} \Gamma_{\mathrm{CAC}}\right)
\end{aligned}
$$




$$
\Delta \mathrm{G}_{\mathrm{ads}}^{0}=\mathrm{nRT} \ln \left(\mathrm{C}_{\Pi} / 55.5\right)-6.022 \Pi_{\mathrm{CAC}} \mathrm{A}_{\mathrm{CAC}}
$$

$$
\Pi_{\mathrm{CAC}}=\gamma_{0}-\gamma_{\mathrm{CAC}}
$$

where, $\mathrm{n}=2$, $\mathrm{R}$ is gas constant $\left(8.314 \mathrm{~J} \cdot \mathrm{mol}^{-1} \cdot \mathrm{K}^{-1}\right)$, $\mathrm{T}$ is absolute temperature in $\mathrm{K}, \gamma$ is surface tension in $\mathrm{mN} / \mathrm{m}, \mathrm{C}$ is concentration in $\mathrm{mol} / \mathrm{L}, \Gamma_{\mathrm{CAC}}$ is expressed in $\mathrm{mol} / \mathrm{m}^{2}$ obtained from the linear portion of the surface tension versus $\log C$ graph. Meanwhile, $\mathrm{N}_{\mathrm{A}}$ is Avogadro's number, from the surface excess concentration, the area/molecule at the interface $A_{C A C}$ is calculated, $\gamma_{0}$ is the surface tension of water, $\gamma_{\mathrm{CAC}}$ is the surface tension at $\mathrm{CAC}, C_{\Pi}$ is the molar concentration of the surfactant in the aqueous phase at a surface pressure $\Pi$ in $\mathrm{mN} / \mathrm{m}$.

The surface areas per molecule of the four systems are about $50 \AA^{2}$, which agrees with the result of other silicone surfactants. In general, for both the known ionic and nonionic surfactants present, the value of the surface area per molecule appears to be determined by the area packed by the hydrated hydrophilic groups. Thus, it was expected that increasing the number of APGE will increase the area per molecule of the surfactant at the interface, but the result was not in agreement with our expectation. This may be due to that increasing the number of APGE could enhance intermolecular interactions and therefore expose polysiloxane chain more to water. Furthermore, exposing polysiloxane chain more to water may influence the formation of aggregates. This may just explain that the CAC decreases with an increase in the numbers of APGE groups. Meanwhile, as we can see, the standard free energy of micellization $\left(\Delta \mathrm{G}_{\text {mic }}^{0}\right)$ and adsorption $\left(\Delta \mathrm{G}_{\text {ads }}^{0}\right)$ are negative, showing the tendencies to form micelles 
in solution and adsorb at the air/water interface. In this case, $\Delta \mathrm{G}_{\text {ads }}^{0}$ is more negative than the corresponding $\Delta \mathrm{G}_{\text {mic }}^{0}$, indicating that adsorption is the predominant event as oppose to aggregation. However, the variation of standard free energy of micellization $\left(\Delta \mathrm{G}_{\text {mic }}^{0}\right)$ and adsorption $\left(\Delta \mathrm{G}^{0}{ }_{\mathrm{ads}}\right)$ along with the variation of APGE is not completely straightforward. This may be attributed to the complexity of their molecular structure.

Table 2 Parameters of aggregation and adsorption at the air/water interface of the APGE-PHMS at $25{ }^{\circ} \mathrm{C}$

\begin{tabular}{|c|c|c|c|c|c|c|c|}
\hline Sample & $\begin{array}{c}\mathrm{CAC} \\
(\mathrm{mol} / \mathrm{L})\end{array}$ & $\begin{array}{c}\gamma_{\mathrm{CAC}} \\
(\mathrm{mN} / \mathrm{m})\end{array}$ & $\begin{array}{l}10^{6} \Gamma_{\mathrm{CAC}} \\
\left(\mathrm{mol} / \mathrm{m}^{2}\right)\end{array}$ & $\begin{array}{c}\mathbf{A}_{\mathbf{C A C}} \\
\left(\AA^{\mathbf{2}}\right)\end{array}$ & $\begin{array}{c}\Pi_{\mathrm{CAC}} \\
(\mathrm{mN} / \mathrm{m})\end{array}$ & $\begin{array}{c}\Delta G_{\text {mic }}^{0} \\
(\mathrm{KJ} / \mathrm{mol})\end{array}$ & $\begin{array}{c}\Delta \mathrm{G}_{\text {ads }}^{0} \\
(\mathrm{KJ} / \mathrm{mol})\end{array}$ \\
\hline $\begin{array}{l}\text { APGE(4) } \\
\text {-PHMS }\end{array}$ & $7.41 \times 10^{-5}$ & 28.80 & 3.50 & 46.00 & 43.30 & -67.03 & -79.02 \\
\hline $\begin{array}{l}\text { APGE(5) } \\
\text {-PHMS }\end{array}$ & $5.25 \times 10^{-5}$ & 25.73 & 2.84 & 56.62 & 46.37 & -68.73 & -84.54 \\
\hline $\begin{array}{l}\text { APGE(6) } \\
\text {-PHMS }\end{array}$ & $4.07 \times 10^{-5}$ & 23.68 & 3.46 & 46.46 & 48.42 & -70.00 & -83.54 \\
\hline $\begin{array}{l}\text { APGE(8) } \\
\text {-PHMS }\end{array}$ & $2.40 \times 10^{-5}$ & 21.76 & 3.36 & 47.78 & 50.34 & -72.61 & -87.09 \\
\hline
\end{tabular}

\subsection{Dynamic Adsorption Property}

Dynamic surface tension measurements performed by the maximum bubble-pressure technique are well suited for researching the adsorption property of surfactant at the air/water interface. Fig.7 shows the curves of the surface tension as a function of the surface age for APGE-PHMS. Obviously, with increasing of the number of APGE, the final surface tension as well as the time required to reach adsorption equilibrium decrease regularly. The consequence is consistent with the 
result of the equilibrium surface tension approximately. This suggests that APGE-PHMS with more APGE groups diffuse faster and absorb quicker at air/water interface from the interior of the solution. In general, the "motion ability" of diffusion and adsorption is caused by the hydrophobic chains. This may be also due to that increasing the number of APGE could improve intermolecular interactions and therefore the polysiloxane chain would be exposed more to water[23-24].

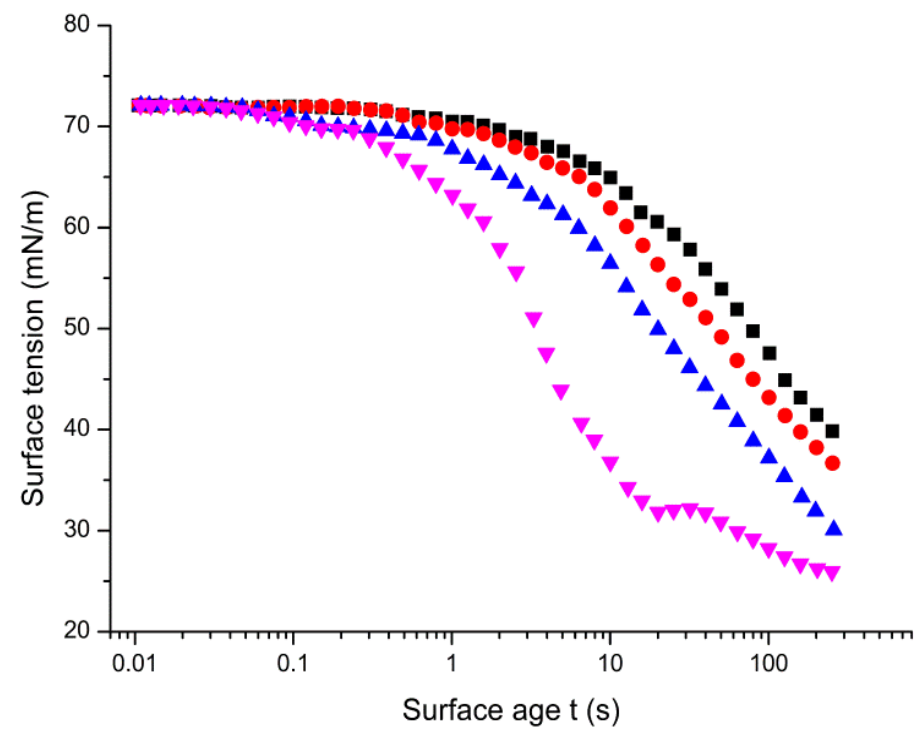

Fig.7 Variation in the dynamic surface tension as a function of surface age for APGE-PHMS

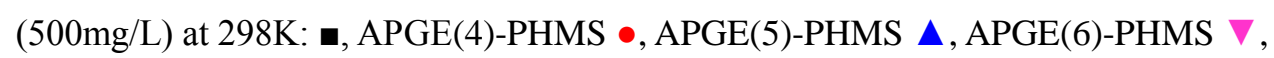
APGE(8)-PHMS

\subsection{Aggregate Property}

In order to visualize the morphology and size of the aggregates for APGE-PHMS solutions above CAC, negative-staining TEM and DLS measurements were carried out.

Spherical assemblies were exhibited in the negative-stained TEM images (Fig. 8). This consequence suggested the presence of aggregates of APGE-PHMS, the diameters of which ranged from 200 to $1000 \mathrm{~nm}$. From the TEM result, one may find 
that these aggregates are much larger in size than the small spherical micelles, which have diameters typically less than $10 \mathrm{~nm}$. The formation of larger complex aggregates is due to the further aggregation of simple assemblies, which is induced by hydrogen bonding or van der Waals interactions among the hydrophilic shells[25-26].
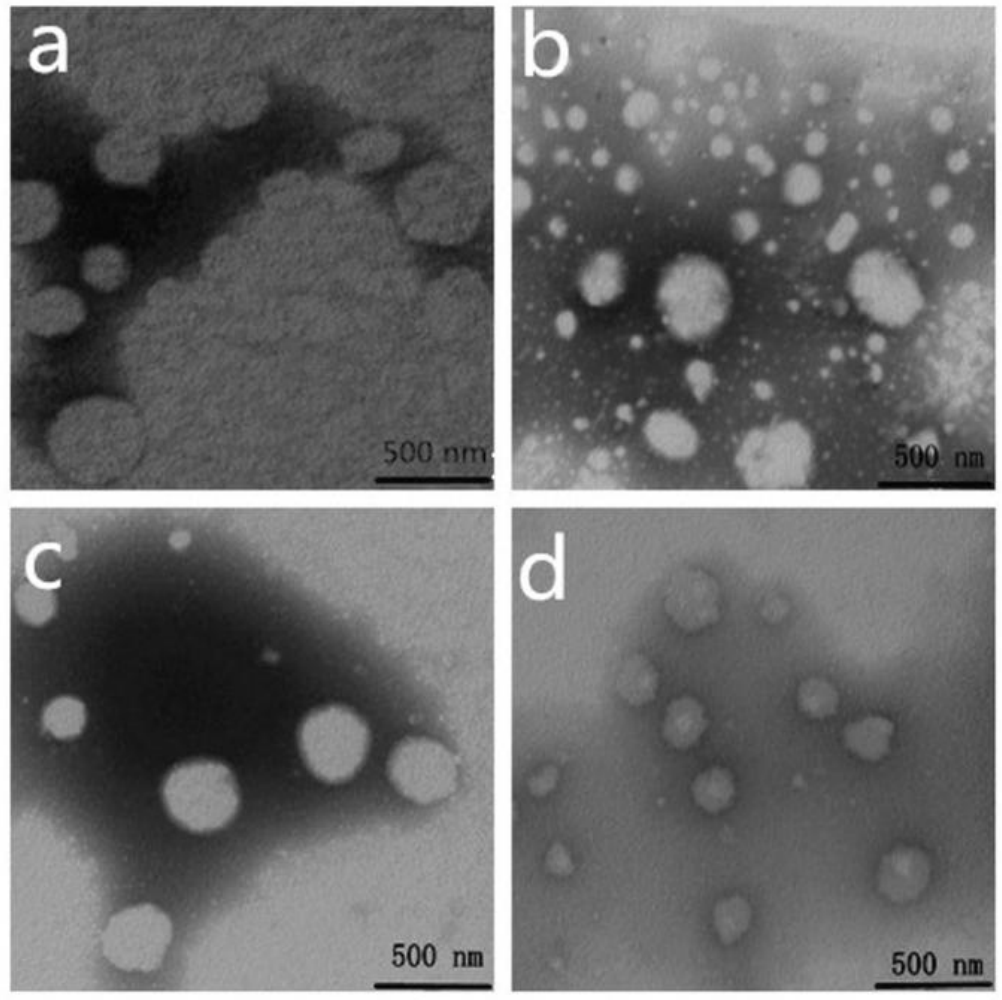

Fig. 8 Negative-stained TEM images of aggregates formed in APGE-PHMS (500mg/L): a, APGE(4)-PHMS b, APGE(5)-PHMS c, APGE(6)-PHMS d, APGE(8)-PHMS

In order to obtain bulk information on average aggregates size and further confirm the presence of aggregates, DLS was performed[22]. The DLS data of the four systems were shown in Fig.10a-d. Obviously, the size of aggregates observed by DLS was in consistent with the data obtained from TEM. One may discover that with an increase in number of hydrophilic APGE groups, the diameter decreases. This might be attributed to an improved difficulty in association of molecules which leads to the diameter decrease[27-29]. 


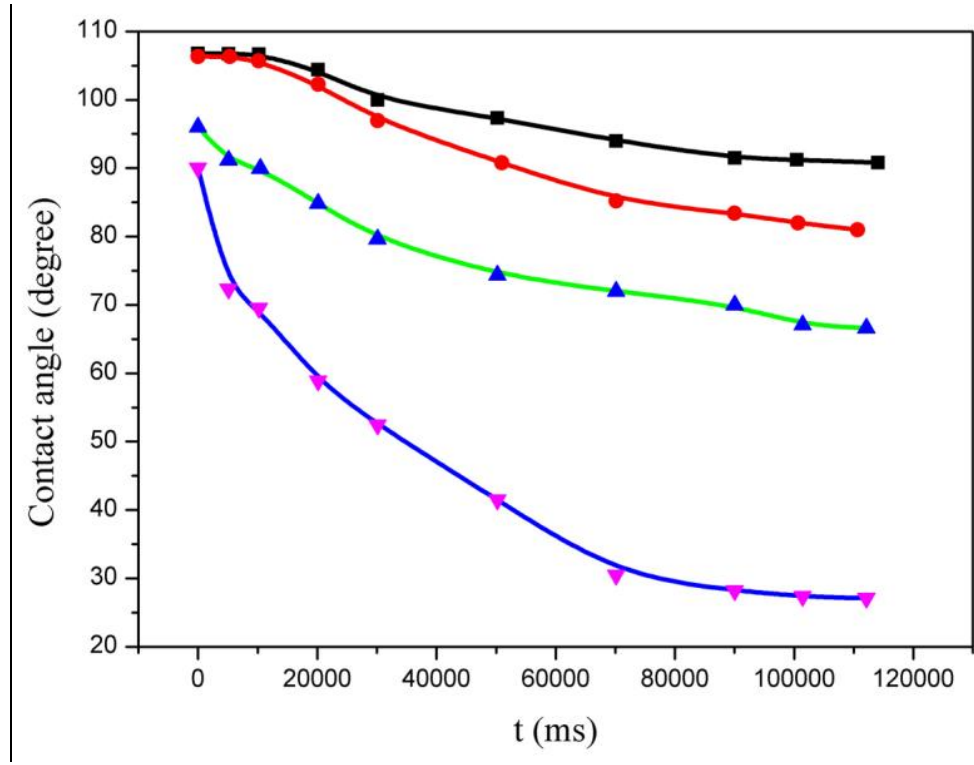

Fig.10 Evolution of dynamic contact angle of droplets of aqueous APGE-PHMS (500mg/L): APGE(4)-PHMS •, APGE(5)-PHMS $\boldsymbol{\Lambda}$, APGE(6)-PHMS $\nabla$, APGE(8)-PHMS

\section{Conclusions}

Polyglycerol-modified polysiloxane were synthesized via the hydrosilylation reaction by grafting polyglycerol groups to the polysiloxane backbone using a two-step method. A series of APGE-PHMS systems with 2, 4, 5, 6 and 8 APGE groups were obtained. Furthermore, solubility, surface activity, adsorption, aggregation and spreading properties of these products were investigated. Measurement on solubility of APGE-PHMS indicated a positive correlation between the number of hydrophilic groups (APGE) and the polarity of suitable solvents. Therefore, , polarity and thus solubility could be modulated by grafting different number of polar polyglycerol groups. Second, the equilibrium surface tension of APGE-PHMS could reach $21 \mathrm{mN} / \mathrm{m}$, which agreed with other silicone surfactants. The system with more APGE groups possessed lower CAC, which might be due to the intermolecular interactions. Third, from dynamic surface tension experiments, we found that the time needed to reach adsorption equilibrium decreased systematically 
with the increase in the hydrophilic groups. Forth, the aggregates of these polymers formed in aqueous solutions could be assigned as spherical assemblies from TEM and DLS analysis. Finally, it was found from contact angle measurements that systems with more APGE groups exhibited better spreading ability. Therefore, given their excellent performance, further investigation of APGE-PHMS will be done in the future work.

\section{Acknowledgments}

This work was financially supported by the National Science Foundation of China (Grant No. 21103228), the National Science \& Technology Pillar Program during the Twelfth Five-year Plan Period (Grant No. 2014BAE03B03) and the Shanxi National Science Foundation of China (Grant No. 2114011014-1 and 201605D211008).

\section{References}

[1] P. Boehm, M. Mondeshki, H. Frey, Macromolecular rapid communications 33 (2012) 1861.

[2] P. Böhm, T.J. Menke, H. Frey, Functional Silicones and Silicone-Containing Block Copolymers (2012) 44-65.

[3] G.Y. Wang, P. Li, Z.P. Du, W.X. Wang, G. Li, Langmuir 31 (2015) 8235.

[4] G. Jonas, R. Stadler, Die Makromolekulare Chemie, Rapid Communications 12 (1991) 625.

[5] G. Jonas, R. Stadler, Acta polymerica 45 (1994) 14.

[6] K. Loos, G. Jonas, R. Stadler, Macromolecular Chemistry and Physics 202 (2001) 3210.

[7] X. Zeng, H.Wang, Y. Chen, et al. Journal of Surfactants and Detergents, 18(2015) 1089-1094..

[8] Y. Zheng, K.J. Thurecht, W. Wang, Journal of Polymer Science Part A: Polymer Chemistry 50 (2012) 629.

[9] J. Chojnowski, M. Cypryk, W. Fortuniak, M. Scibiorek, K. Rozga-Wijas, Macromolecules 36 (2003) 3890.

[10] J.K. Paulasaari, W.P. Weber, Macromolecules 33 (2000) 2005.

[11] V. Bellas, H. Iatrou, N. Hadjichristidis, Macromolecules 33 (2000) 6993.

[12] Y. Hou, C.A. Schoener, K.R. Regan, Biomacromolecules 11 (2010) 648.

[13] B. Radi, R.M. Wellard, G.A. George, Macromolecules 43 (2010) 9957. 
[14] F. Gonzaga, G. Yu, M.A. Brook, Macromolecules 42 (2009) 9220.

[15] G.Y. Wang, Z.P. Du, W. Zhang, Q.Y. Cao, Tenside Surfactants Detergents 46 (2009) 214

[16] G.Y. Wang, W.S. Qu, Z.P. Du, et al. The Journal of Physical Chemistry B, 117(2013) 3154-3160.

[17] G.Y. Wang, Z.P. Du, Q.X. Li, W. Zhang, The Journal of Physical Chemistry B 114 (2010) 6872.

[18] K. Isobe, S. Okazaki, Novel organopolysiloxane having alcoholic hydroxy groups and a method for the preparation thereof. US Patents (4431789), 1984.

[19] D.w. Chung, J.C. Lim, Colloids and Surfaces A: Physicochemical and Engineering Aspects 336 (2009) 35.

[20] G.Y. Wang, X. Li, Z.P. Du, E.Z. Li, P. Li, Journal of Molecular Liquids 197 (2014) 197.

[21] Z.P. Du, L. Wang, G.Y. Wang, S. Wang, Colloids and Surfaces A: Physicochemical and Engineering Aspects 381 (2011) 55.

[22] F. Han, G. Zhang, Colloids \& Surfaces A Physicochemical \& Engineering Aspects 237 (2004) 79.

[23] Z.L. Peng, C.F. Lu, M.L. Xu, Journal of surfactants and detergents, (12)2009 331-336.

[24] J. Eastoe, J.S. Dalton, Advances in Colloid Interface Sci., 85(2000) 103-144.

[25] Y. Tomokazu, O. Akiko, E. Kunio,. Langmuir, 22(2006) 4643-4648.

[26] Z.L. Peng, Q. Wu, T. Cai, H. Gao, K. Chen, Colloids and Surfaces A: Physicochemical and Engineering Aspects, 342(2009) 127-131.

[27] Z.P. Du, E.Z. Li, Y. Cao, X. Li, G.Y. Wang, Colloids and Surfaces A: Physicochemical and Engineering Aspects 441 (2014) 744.

[28] H.C. Zhang, Z.N. Liu, et al. Carbohydrate Research, 345(2010) 87-96.

[29] Y. Zheng, K. J. Thurecht, W. Wang, Journal of Polymer Science Part A: Polymer Chemistry, 50 (2012) 629-637.

[30] N. Iwakiri, T. Nishikawa, Y. Kaneko, et al. Colloid Poly. Sci., 287(2009) 577-582.

[31] Y. Shen, A. Couzis, J. Koplik, et al. Langmuir, 21(2005) 12160-12170.

[32] D.W. Chung, J.C Lim. Colloids and Surfaces A: Physicochemical and Engineering Aspects, 336(2009) 35-40. 

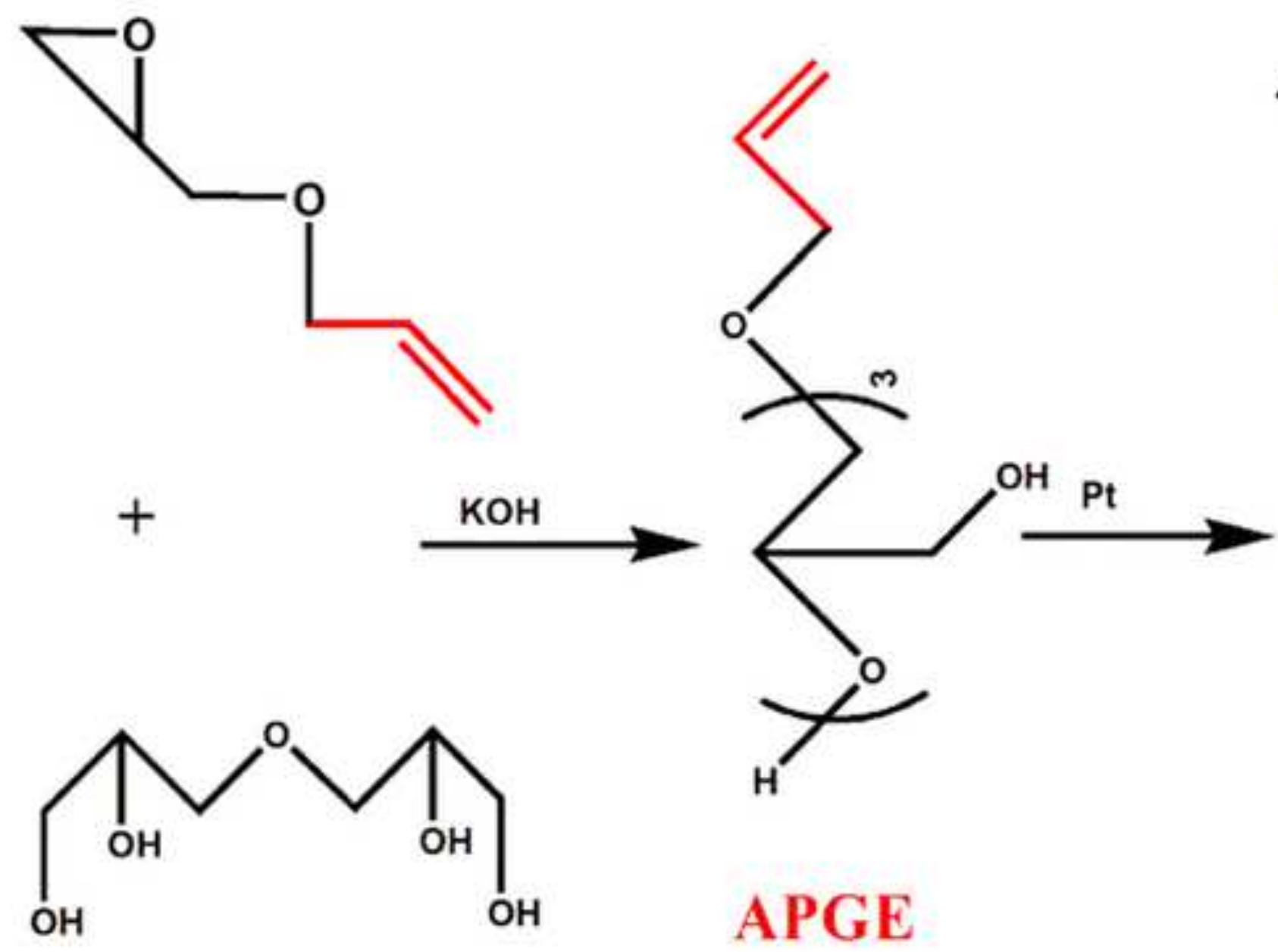

siloxane bakebone

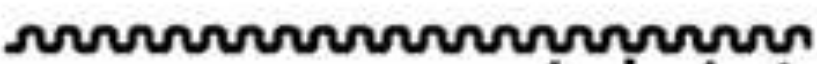
$\downarrow \downarrow \downarrow \downarrow$

G G G G

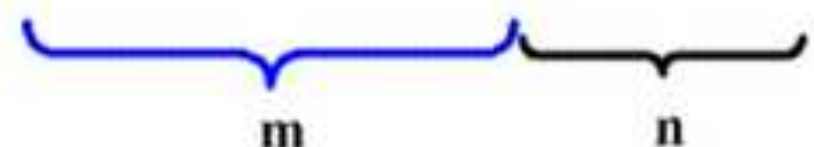

(1) $\mathrm{m}=8, \mathrm{n}=2$

(2) $m=6, n=4$

(3) $m=5, n=5$

(4) $m=4, n=6$

(5) $\mathrm{m}=2, \mathrm{n}=8$

APGE-PHMS 